Kharangarh PR, Umapathy S, Singh G, Sharma RK and Kumar A (2017) High-performance pseudocapacitor electrode materials: cobalt (II) chloride-GQDs electrodes. Emerging Materials Research 6(2): 227-233,
Emerging Materials Research Volume 6 Issue EMR2 Composite materials research article Received 24/10/2016 Accepted 14/06/2017 Published online 11/07/2017

Keywords: quantum dots/raman spectroscopy/supercapacitors

\title{
High-performance pseudocapacitor electrode materials: cobalt (II) chloride-GQDs electrodes
}

Poonam Rani Kharangarh PhD*

Postdoctoral Fellow, Department of Chemistry, University of Delhi, Delhi, 110007, India

Siva Umapathy PhD

Professor, Department of Inorganic and Physical Chemistry, Indian Institute of Science, Bangalore, India
Gurmeet Singh PhD

Professor, Department of Chemistry, University of Delhi, Delhi, India

Raj Kishore Sharma PhD

Associate Professor, Department of Chemistry, University of Delhi, Delhi, India

Akshay Kumar MSC

PhD student, Department of Chemistry, University of Delhi, Delhi, India

This paper reports an effective synthesis, for the first time, of cobalt (II) chloride $\left(\mathrm{CoCl}_{2}\right)$-doped graphene quantum dots (cobalt (II) chloride-GQDs). Excellent results were obtained by using cobalt (II) chloride powder and graphene oxide as source materials in a facile hydrothermal process. The prepared materials were characterized by using highresolution transmission electron microscopy (HRTEM), Raman spectroscopy, ultraviolet-visible spectroscopy, Fourier transform infrared spectroscopy, powder X-ray diffraction and photoluminescence (PL). HRTEM images suggest that the diameter of the majority of the cobalt (II) chloride-GQDs is in the narrow range of 2.5-7 nm, much smaller than that of GQDs, which are approximately $15 \mathrm{~nm}$. By using different excitation energies in $\mathrm{PL}$, the appearance of a peak introduces an additional energy level between $\pi$ and $\pi^{*}$, reinforcing the cobalt (II) chloride doping process. The authors observed that cobalt (II) chloride electrodes can exhibit a reversible redox reaction of $\mathrm{Co}^{2+} \leftrightarrow \mathrm{Co}^{4+}$ and show specific pseudocapacitance of $\sim 300 \mathrm{~F} / \mathrm{g}$. The new charge storage mechanism using cobalt (II) chloride electrodes is easily scalable and highly economical for future pseudocapacitors and can be extended to a large variety of commercial inorganic salt electrodes.

\section{Notation}

C specific capacitance (C) of cobalt (II) chloride-GQDs

$d_{\mathrm{v}} / d_{\mathrm{t}} \quad$ obtained from the discharge curve

I constant discharge current

$m$ mass of active materials within the electrode

\section{Introduction}

Supercapacitors or ultracapacitors have attracted recent attention due to their high power density, high charge-discharge rates and long cycle-life performance. ${ }^{1-3}$ The most challenging work is to be able to achieve a high energy storage capacity in pseudocapacitance to control its specific surface area and pore size for enhancing the electrical conductivity compared to that of electric double-layer capacitors. Due to the poor electrical conductivity of the pseudocapacitive electrodes, the limitations of faradic reactions lead to unsatisfactory electrochemical performance and life cycles.

Graphene is considered as one of the most promising electrode materials ${ }^{4}$ of supercapacitors due to its unique structure and properties, such as large surface area and high conductivity. A significant amount of work has been performed on novel capacitors, including microsupercapacitors, ${ }^{5,6}$ solid-state supercapacitors ${ }^{7,8}$ and wearable supercapacitors. ${ }^{9,10} \mathrm{Li}$ et al. ${ }^{11}$ reported the electrochemical preparation of graphene quantum dots (GQDs) in $0 \cdot 1 \mathrm{M}$ phosphatebuffered solution (PBS) with a filtration-formed graphene film as the working electrode (WE) upon the application of cyclic voltammetry (CV) scan. Later in 2011, the same group used nitrogen (N)-containing tetrabutylammonium perchlorate in acetonitrile as the electrolyte instead of PBS. Zhang et al., ${ }^{12}$ in 2012, prepared GQDs by electrolysis of a graphite rod in alkaline condition of $0 \cdot 1 \mathrm{M}$ sodium hydroxide $(\mathrm{NaOH})$ and observed yellow luminescent GQDs with an average diameter of 5-10 nm, mostly consisting of a single graphene layer, but the condition of roomtemperature reduction is critical to form the yellow luminescent GQDs. Liu et $a l^{5}$ fabricated GQDs//manganese oxide $\left(\mathrm{MnO}_{2}\right)$ asymmetric supercapacitor in aqueous electrolyte, which shows an excellent rate capability, higher specific capacitance $\left(1107 \cdot 4 \mu \mathrm{F} / \mathrm{cm}^{2}\right)$ and higher energy density $(0 \cdot 154 \mu \mathrm{Wh} / \mathrm{cm})$ as compared to GQDs//GQDs symmetric microsupercapacitors. Zhang et $a l^{8}{ }^{8}$ proposed that GQDs can serve as solution- and solid-type electrolytes for supercapacitors because of the complete ionization of weak acidic oxygen-bearing functional groups after neutralization. The major limitation of supercapacitors is their low energy density compared to that of battery systems. Ji et al. ${ }^{13}$ developed a novel in situ activation method for the fabrication of a three-dimensional free-standing nitrogen-doped porous graphene/ graphite foam with a high capacity of $102 \mathrm{mAh} / \mathrm{g}$ and a charging time of $\sim 90 \mathrm{~s}$ for the use of a high-rate lithium ion battery anode. Chen et al., ${ }^{14}$ in 2016, investigated the role of topological defects and nitrogen dopants on the electrical double-layer capacitance for designing better supercapacitors. Parlak et al., ${ }^{15}$ in 2017, showed that porosity leads to significantly high specific capacitance $(640 \mathrm{~F} / \mathrm{g})$ with high energy $(14 \cdot 2 \mathrm{Wh} / \mathrm{kg})$ and power densities $\left(9.67 \times 10^{3} \mathrm{~W} / \mathrm{kg}\right)$. 
Detailed work needs to be performed to make improvements in the electrochemical performance of pseudocapacitors. The most investigated materials, such as nickel (II) oxide (NiO), nickel (II) hydroxide $\left(\mathrm{Ni}(\mathrm{OH})_{2}\right)$, manganese oxide, cobalt (II,III) oxide $\left(\mathrm{Co}_{3} \mathrm{O}_{4}\right)$, copper (II) chloride $\left(\mathrm{Co}(\mathrm{OH})_{2}\right)$ and copper (II) chloride $\left(\mathrm{CuCl}_{2}\right)$, have been reported so far. ${ }^{16-20}$ However, commercial water-soluble inorganic salts, with multiple valence state metal ions, have not been explored in aqueous electrolytes for their pseudocapacitance.

The commercial water-soluble inorganic salt cobalt (II) chloride $\left(\mathrm{CoCl}_{2}\right)$ was the best apposite transition metal to be doped with graphene oxide (GO) or GQDs. In this work, initially, the synthesized GQDs were used as they extend some functional groups after the hydrothermal process. Subsequently, cobalt (II) chloridedoped GQDs (cobalt (II) chloride-GQDs) were synthesized. To the best of the authors' knowledge, they believe that this paper reports the synthesis for the first time of cobalt (II) chloride-GQDs, in the literature, along with their morphology and optical properties. The authors have demonstrated that cobalt (II) chloride-GQD electrodes can exhibit a fast and reversible redox reaction of $\mathrm{Co}^{2+} \leftrightarrow \mathrm{Co}^{4+}$ and deliver very high specific pseudocapacitance of $300 \mathrm{~F} / \mathrm{g}$. The obtained results reflect the view that the synthesis of advanced materials in GQDs is an important step to increase the specific capacitance and current density of inorganic pseudocapacitors.

\section{Experimental details}

\subsection{Synthesis of GO/GQDs/cobalt (II) chloride-doped GQDs}

Graphite oxide was prepared by using commercially available graphite powder following the Hummers and Offemann method. ${ }^{21,22}$ For the preparation of cobalt (II) chloride-GQDs, $5.95 \mathrm{mg}$ of cobalt (II) chloride hexahydrate $\left(\mathrm{CoCl}_{2} \cdot 6 \mathrm{H}_{2} \mathrm{O}\right)$ was dissolved in $2 \mathrm{ml}$ of hydrochloric acid. The solution was added into $40 \mathrm{ml}$ of aqueous solution with $2 \mathrm{ml}$ of GQDs prepared under magnetic stirring. Here, GQDs were also prepared using the hydrothermal method except for the addition of cobalt (II) chloride hexahydrate for comparison. The $\mathrm{pH}$ was adjusted to 12 with potassium hydroxide $(\mathrm{KOH})$, and the mixture was transferred to Teflon-lined autoclave and heated at $150^{\circ} \mathrm{C}$ for $4 \mathrm{~h}$. This solution was naturally cooled to room temperature, and subsequently, the cobalt (II) chloride-doped GQDs were collected by filtration through a $0 \cdot 22 \mu \mathrm{m}$ Teflon membrane. The final resulting solution was then centrifuged at 4000 revolutions per minute before further characterization was carried out.

\subsection{Electrochemical measurements}

Electrochemical measurements were performed using a CHI-760C potentiostat-galvanostat instrument. CV curves were measured in a $7.6 \mathrm{~g} / 1$ potassium chloride $(\mathrm{KCl})$ solution in a standard threeelectrode cell with a silver $(\mathrm{Ag}) /$ silver chloride $(\mathrm{AgCl})$ as a reference electrode $(\mathrm{RE})$ and platinum $(\mathrm{Pt})$ as a counter electrode (CE), respectively. The WE was prepared by drop-casting of a cobalt (II) chloride-GQD solution onto a glassy carbon electrode. The specific capacitance $C(\mathrm{~F} / \mathrm{g})$ was calculated by using the equations by Kumar et al., ${ }^{23}$ Bard and Faulkner, ${ }^{24}$ Du et al. ${ }^{25}$ and $\mathrm{Ye}$ et al. $^{26}$ Electrochemical impedance spectroscopic measurements were employed to investigate the ohmic resistance of the electrode system, which is due to the electrolyte and the cell components and charge transfer resistance.

\subsection{Material characterization}

Raman spectra were obtained by using a Renishaw spectrometer by way of a microscope system equipped with an argon ion $\left(\mathrm{Ar}^{+}\right)$laser $(\lambda=514.5 \mathrm{~nm})$. Ultraviolet (UV)-visible (Vis) absorption spectra of dispersions were recorded on a PerkinElmer Lambda 35 spectrophotometer, with a slit width of $2 \mathrm{~nm}$ and scan speed of $240 \mathrm{~nm} / \mathrm{min}$. The presence of functional groups was confirmed by Fourier transform infrared (FTIR) spectra of samples by using a PerkinElmer Spectrum-Two spectrometer with attenuated total reflectance and specular reflectance operating with a resolution of $4 \mathrm{~cm}^{-1}$ in the frequency range of $400-4000 \mathrm{~cm}^{-1}$. Powder X-ray diffraction (PXRD) patterns were recorded using a Bruker D8 Advance X-ray diffractometer $(40 \mathrm{kV}, 50 \mathrm{~mA}$, sealed copper $(\mathrm{Cu})$ $\mathrm{X}$-ray tube) equipped with a graphite monochromator. Transmission electron microscope images were obtained using an FEI Technai G2 20 electron microscope operating at $200 \mathrm{kV}$. Photoluminescence (PL) spectroscopic measurements for excitation and emission spectra were recorded to investigate the energy levels in cobalt (II) chloride-GQDs and GQDs.

\section{Results and discussion}

Figures 1(a) and 1(b) show the high-resolution transmission electron microscope (HRTEM) images of the GQDs and cobalt (II) chloride-GQDs, respectively. The majority of the cobalt (II) chloride-GQDs are estimated to be in the narrow range of 2.5-7 nm dia., which is much smaller than the size of GQDs (around $15 \mathrm{~nm}$ ), synthesized by cutting GO sheets with a modified Hummers oxidation cutting methods. The selected area electron diffraction pattern is shown in the inset of Figure 1(b). The bright points correspond to the planes of the quantum structures (cobalt (II) chloride-GQDs) with a spacing of $0 \cdot 156 \mathrm{~nm}$. This reveals the transformation of amorphous quantum dots to highly crystalline cobalt (II) chloride-GQDs. These are consistent with the (111), (200), (220) and (311) crystal planes of face-centered cubic structure of cobalt $(\mathrm{Co})^{27}$ in cobalt (II) chloride-GQDs. The C-C bonds with a length $0 \cdot 154 \mathrm{~nm}^{28}$ are located on the sheet planes, whereas a $d$-spacing of $0 \cdot 156 \mathrm{~nm}$ signifies the intersheet distance. This corresponds to the absence of ordering in the longitudinal direction. Otherwise, a sharp ring would have appeared corresponding to the $d$-spacing, which is larger than the $\mathrm{C}-\mathrm{C}$ bond length.

In order to investigate the high degree of crystallinity of cobalt (II) chloride-doped GQDs, PXRD measurements were carried out (Figure 2). After oxidation, a typical strong and broad peak near $10 \cdot 4^{\circ}$ is observed for GO, which is assigned to the (002) plane. The value corresponds to an interlayer distance of $11.45 \AA$, indicating a remarkable expansion of graphite due to the presence of oxygen containing functional groups on both sides of graphene sheets and atomic scale roughness arising from $\mathrm{sp}^{3}$ bonding in carbon. ${ }^{29,30}$ The 


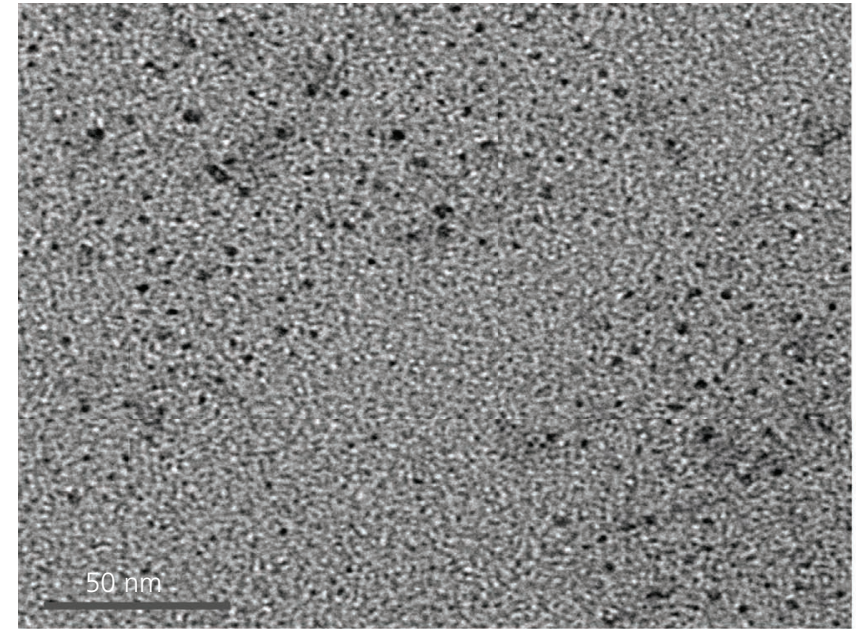

(a)

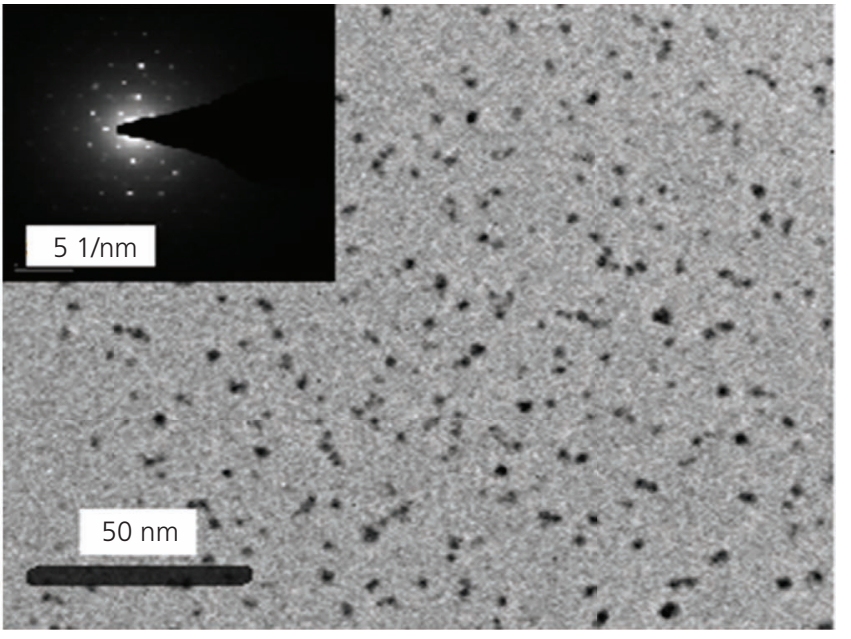

(b)

Figure 1. HRTEM images of (a) GQDs and (b) cobalt (II) chloride-GQDs

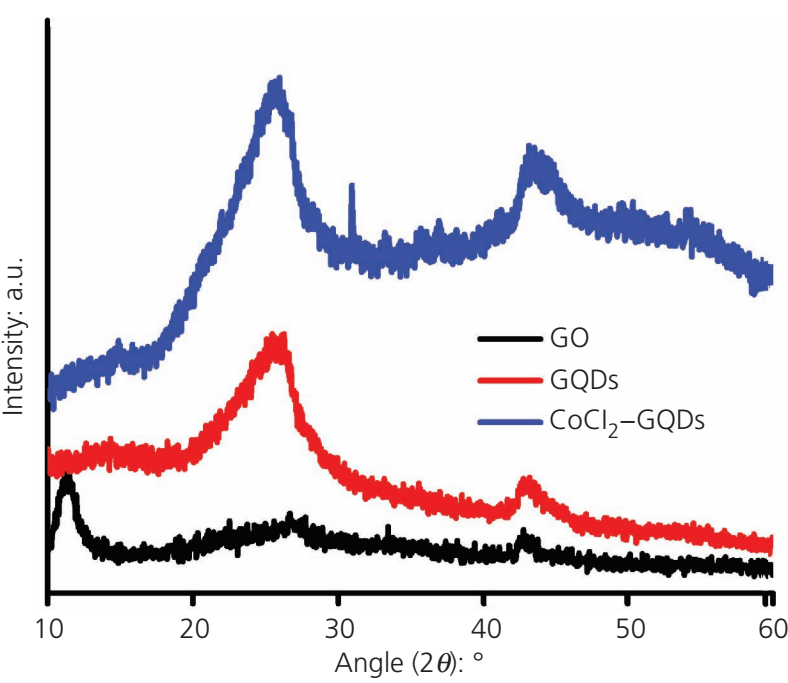

Figure 2. PXRD patterns of GO, GQDs and cobalt (II) chloride-GQDs

disappearance of a sharp peak at $11.45 \AA$ and the shift of the (002) reflection, after hydrothermal treatment, suggests the conversion of GO to GQDs. The (002) reflection of GQDs is present at $25 \cdot 54^{\circ} .31$ The observed broad, low-intensity (002) reflection is possibly due to the broken regular stacking and reduction in size. The XRD pattern of cobalt (II) chloride-GQDs, on the other hand, consists of strong reflections $(2 \theta)$ at $25 \cdot 8$ and $43 \cdot 6^{\circ}$, which are assigned to the (111) and (311) planes, respectively, to a face-centered cubic structure of the cobalt metal, which is in good agreement with the previous reports on metallic cobalt. ${ }^{27,32}$

Figure 3 depicts the FTIR spectra of the as-prepared GO, GQDs and cobalt (II) chloride-GQDs. The FTIR spectra of pristine GO (shown in Figure 3 ) shows the presence of $\mathrm{C}-\mathrm{O}\left(v_{\mathrm{C}-\mathrm{O}}\right.$ at $\left.1040 \mathrm{~cm}^{-1}\right), \mathrm{C}-\mathrm{OH}\left(v_{\mathrm{C}-\mathrm{O}}\right.$ at $\left.1389 \mathrm{~cm}^{-1}\right), \mathrm{C}=\mathrm{O}$ in carboxylic acid and carbonyl moieties $\left(v_{\mathrm{C}-\mathrm{O}}\right.$ at 1639 and $\left.1726 \mathrm{~cm}^{-1}\right)$. After hydrothermal treatment of GO (or in GQDs), the $\left.v_{\mathrm{C}-\mathrm{O}}\right)$ band disappeared, further confirming the removal of oxygen functional groups from GO. In addition, two strong vibrational bands can be seen at 1692 and $1558 \mathrm{~cm}^{-1}$, which are attributed to the $\mathrm{C}=\mathrm{C}$ stretching vibration of unoxidized graphitic $\left(\mathrm{sp}^{2}\right)$ domains. ${ }^{29,30}$ The infrared absorption spectra of cobalt (II) chloride in the $700-1500 \mathrm{~cm}^{-1}$ region has been reported by several researchers. ${ }^{33,34}$ The cobalt (II) chloride-doped GQDs exhibit a characteristic peak between 500 and $1000 \mathrm{~cm}^{-1}$ corresponding to the presence of cobalt (II) ions $\left(\mathrm{Co}^{2+}\right)$ in doped GQDs. ${ }^{35}$ Furthermore, a few strong peaks at 1097 and $1389 \mathrm{~cm}^{-1}$ (C-O carboxyl or carbonyl stretching vibrations or $\mathrm{O}-\mathrm{H}$ deformation in the $\mathrm{C}-\mathrm{OH}$ group) and $1639 \mathrm{~cm}^{-1}$ were seen in cobalt (II) chloride results reported previously. ${ }^{36}$

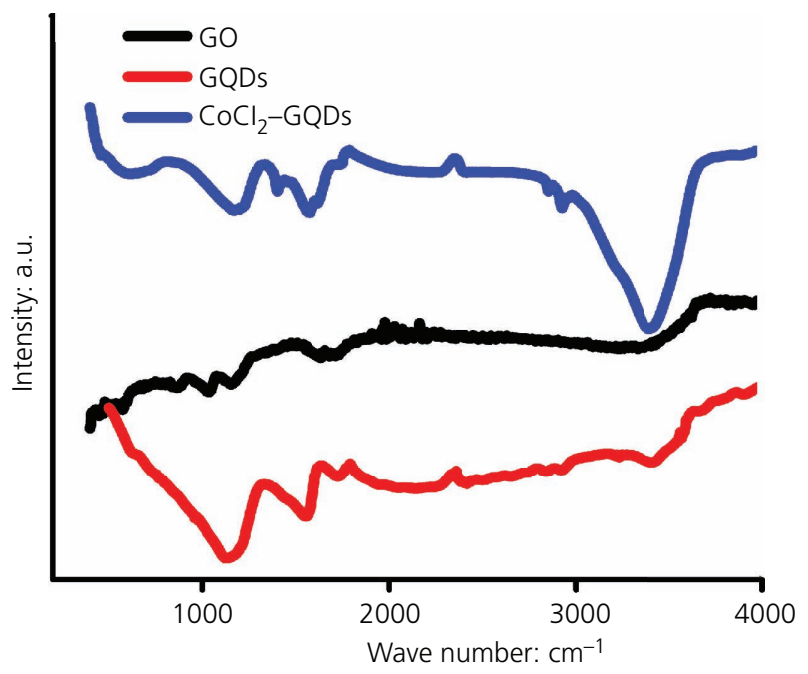

Figure 3. FTIR images of GO, GQDs and cobalt (II) chloride-GQDs 
High-performance pseudocapacitor

electrode materials: cobalt (II)

chloride-GQDs electrodes

Kharangarh, Umapathy, Singh, Sharma and Kumar

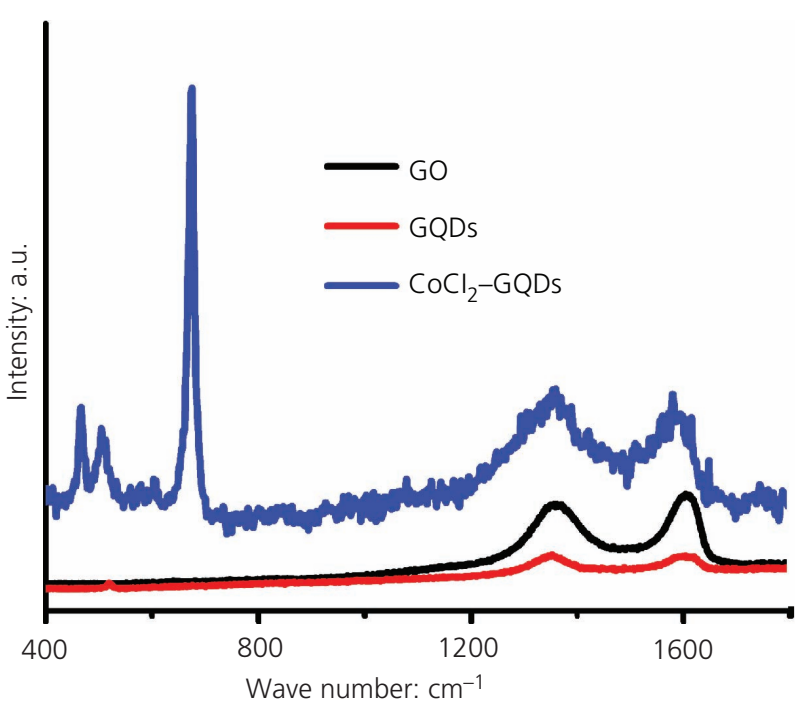

Figure 4. Raman spectra of GO, GQDs and cobalt (II) chloride-GQDs

Figure 4 shows the Raman spectra of GO and GQDs and changes in the GQD structure after doping with cobalt (II) chloride. The ratio of the peak intensities of the $\mathrm{D}$ and $\mathrm{G}$ bands $\left(I_{\mathrm{D}} / I_{\mathrm{G}}\right)$ describes the disordered nature and structural defects of graphitic carbon. $^{37}$ The intensity ratios of GO, GQDs and cobalt (II) chloride-GQDs are $0.91,0.91$ and 0.85 , respectively. The observed intensity ratio of the $\mathrm{D}$ and $\mathrm{G}$ bands of cobalt (II) chloride-GQDs is lower than that of GO and GQDs, suggesting that the intercalation of cobalt (II) atoms into the conjugated carbon has led to more ordered structure. In addition, the peaks at 467,508 and $675 \mathrm{~cm}^{-1}$ are related to the vibrational modes of cobalt (II) ions in cobalt (II) chloride-GQDs.

To investigate the modification of the optical properties of GQDs, before and after doping, UV-Vis absorption spectra and PL spectra of GQD and cobalt (II) chloride-GQD aqueous solutions were acquired. Figure 5(a) shows the UV-Vis absorption spectra of GO, GQDs and cobalt-functionalized GQDs in aqueous solutions. Absorption peaks at 232 and $300 \mathrm{~nm}$ were observed in GO. The spectrum of GQDs is characterized by a $323 \mathrm{~nm}$ band, which is redshifted from $232 \mathrm{~nm}$ of GO. The absorption peak at $323 \mathrm{~nm}$ is a residue of $\mathrm{n}-\pi *$ transitions of the $\mathrm{C}=\mathrm{O}$ bond. ${ }^{38}$ The presence of the $\mathrm{n}$ band is possibly related to oxygen-related functional groups which exist between the valence band ( $\pi$ band) and conduction band ( $\pi^{*}$ band). After doping with cobalt (II) chloride, a new blueshifted peak is observed at $253 \mathrm{~nm}(4.9 \mathrm{eV})$. The absorption peak at $253 \mathrm{~nm}$ is caused by $\pi-\pi^{*}$ transitions of the $\mathrm{C}=\mathrm{C}$ bond. ${ }^{38}$

Figure 5(b) shows the PL spectra of GQDs and cobalt (II) chloride-GQDs under different excitation wavelengths from 300 to $360 \mathrm{~nm}$ with an interval of $20 \mathrm{~nm}$. The results of PL studies demonstrate that the observed spectra from GQDs could correlate with the transition at $435 \mathrm{~nm}$. Nonetheless, a similar trend is observed in the PL emission in GQDs with negligible dependence on the size of the quantum structures. The two electronic transitions at 385 and $435 \mathrm{~nm}$ can be attributed to the transitions from cobalt-related surface states to the $\pi^{*}$ band and oxygenrelated surface states which are common in both GQDs and after doping. Also, different emission peaks are observed in cobalt (II) chloride-GQDs with excitation wavelength in the range of $300-360 \mathrm{~nm}$ due to the dependence on size. The authors believe that due to the presence of cobalt-related functional groups and $\mathrm{C}=\mathrm{C} \mathrm{sp}{ }^{2}$ carbon domains, different electronic transition states will appear due to bonding and antibonding of molecular orbitals. The authors attribute this tunable PL to the observed band-to-band transitions $\left(\pi^{*}-\pi\right.$ transitions $\left.\sim 325 \mathrm{~nm} / 3 \cdot 82 \mathrm{eV}\right)$ resulting from cobalt (II) ion metal groups and interstate band transitions ( $\mathrm{n}-\pi^{*}$ transitions $\sim 385 \mathrm{~nm} / 2 \cdot 85 \mathrm{eV}$ ), which is also observed in GQDs.

\subsection{Electrochemical characterization}

The electrochemical properties of cobalt (II) chloride-GQDs were investigated in a three-electrode cell by using $1 \mathrm{M}$ potassium

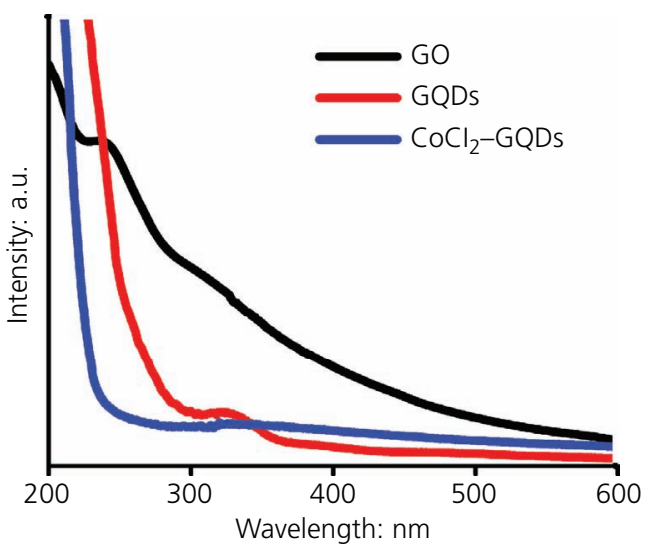

(a)

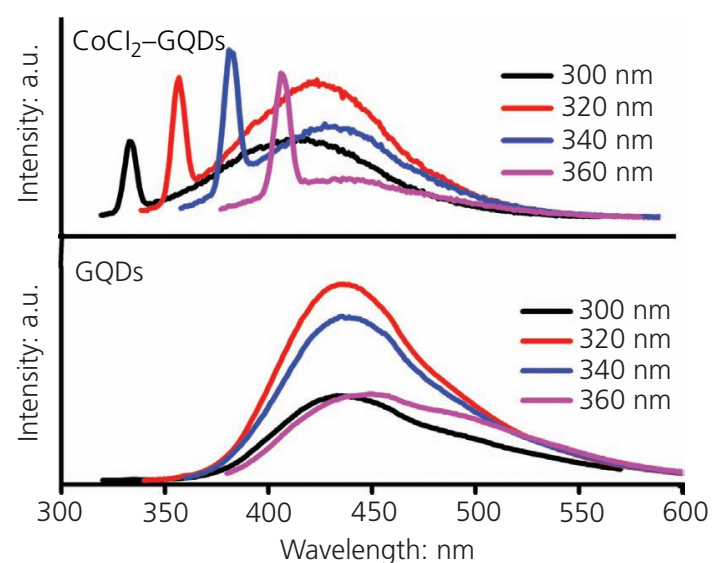

(b)

Figure 5. (a) UV-Vis absorption spectra of GO, GQDs and cobalt (II) chloride-GQDs and (b) PL spectrum of GQDs and cobalt (II) chloride-GQDs with different excitations 


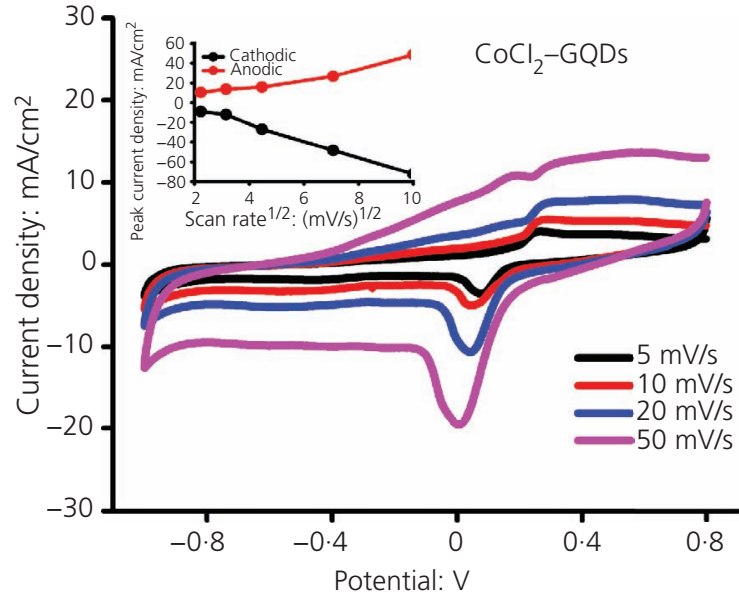

(a)

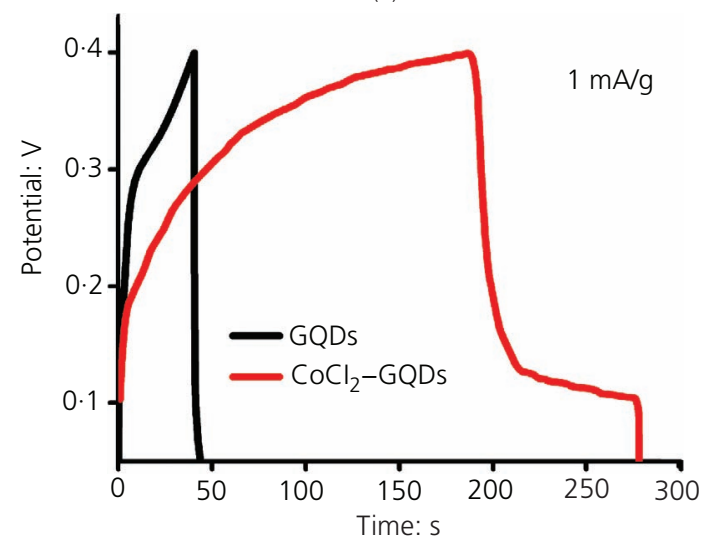

(c)

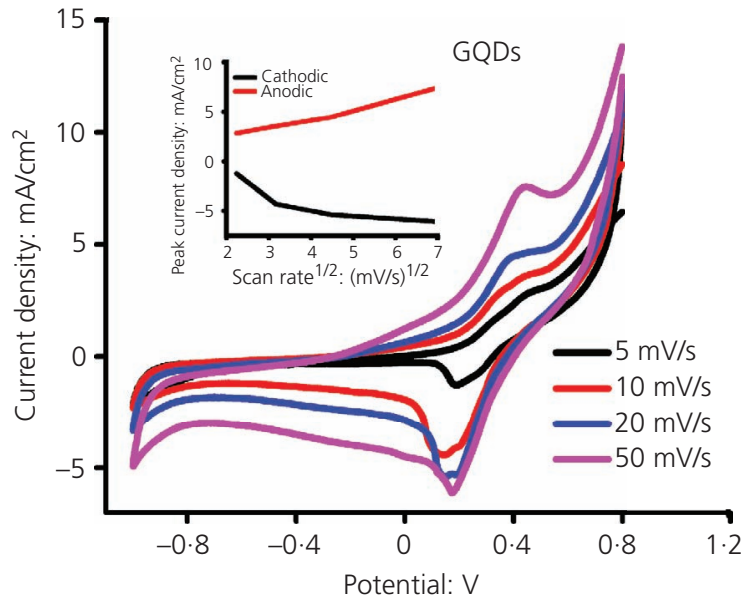

(b)

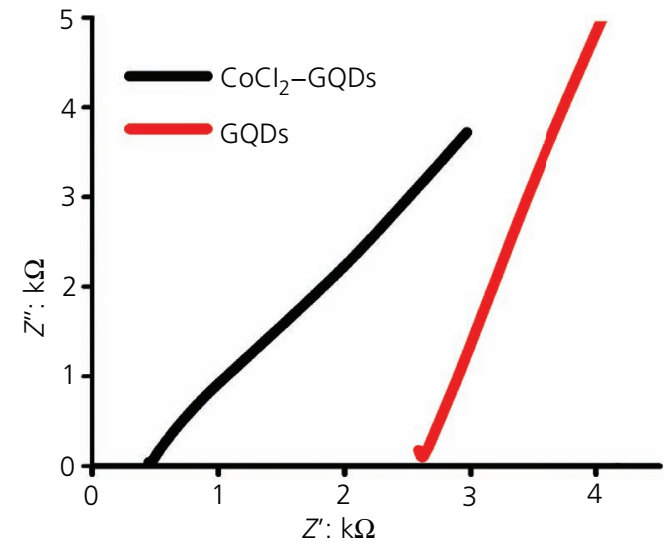

(d)

Figure 6. CV of (a) cobalt (II) chloride-GQDs and (b) GQDs at different scan rates; the inset shows the anodic and cathodic currents against the square root of scan rates for corresponding electrodes. Silver/silver chloride as an RE and platinum wire as a CE were used. (c) Galvanostatic charge-discharge of GQDs and cobalt (II) chloride-GQDs at $1 \mathrm{~mA} / \mathrm{g}$ current density and (d) impedance spectroscopy of GQDs and cobalt (II) chloride-GQDs

chloride electrolyte. CV curves (Figures 6(a) and 6(b)) were plotted to observe the charge storage mechanism of the cobalt (II) chloride-GQD electrode. Cobalt (II) chloride-GQD electrodes in the potassium chloride electrolyte can show a pseudocapacitance behavior in terms of fast and reversible redox reactions.

Figure 6(a) and 6(b) show the cyclic voltammograms of GQDs and cobalt (II) chloride-GQDs. They were recorded from -0.8 to $+1.0 \mathrm{~V}$ with different scan rates of $5,10,20$ and $50 \mathrm{mV} / \mathrm{s}$. This study showed that the inorganic salt cobalt (II) chloride shows a pseudocapacitive characteristic and anomalous specific capacitance (Figure 6(a)). A pair of cathodic and anodic peaks at -0.24 and $0 \cdot 1 \mathrm{~V}$ is observed with different scan rates in GQDs and cobalt (II) chloride-GQDs which show the redox behavior. The anodic peak of the redox pair is responsible for the oxidation of cobalt (II)/ cobalt (IV) $\left(\mathrm{Co}^{4+}\right)$ ions, ${ }^{39}$ whereas the cathodic peak corresponds to a reduction process following the faradic reduction reactions from cobalt (IV) ion to cobalt (II) ion. It is to be noted that the cathodic peaks shift positively and the anodic peaks shift negatively with incremental potential sweep rates, which are mainly due to the resistance of the electrode. ${ }^{40}$ The cathode peak shifts toward more positive potentials is due to the presence of oxygen functionalities. In addition, the current density increases with increase in scan rate, which indicates a good rate capability of the cobalt (II) chloride-GQDs compared to GQDs. The non-rectangular shapes of the $\mathrm{CV}$ curves reveal that the charge storage is a characteristic of the pseudocapacitance process originating from the reversible redox reactions of cations. The plot of the square root of the scan rate of anodic and cathodic peak current (shown in the inset of Figures 6(a) and 6(b)) gives a straight line, indicating that the observed electrochemical behavior is a surface confined phenomenon.

Figure 6(c) shows the galvanostatic charge-discharge curves. The value of the specific capacitance was obtained from the discharge curve in accordance with the following equation ${ }^{40}$

1. $C=\frac{1}{m} \frac{\mathrm{d} t}{\mathrm{~d} V}$ 
where $I$ is the constant discharge current, $m$ is the mass of active materials within the electrode and $\mathrm{d} V / \mathrm{d} t$ can be obtained from the discharge curve. The specific capacitance (C) of cobalt (II) chloride-GQDs is found to be $300 \mathrm{~F} / \mathrm{g}$, which is four times higher than that of GQDs $(C=75 \mathrm{~F} / \mathrm{g})$. The high specific capacitance obtained from the well-known faradic reaction of $\mathrm{Co}^{2+} \leftrightarrow \mathrm{Co}^{4+}$ originates from the electrochemical reactions on the cobalt (II) chloride electrode due to the existence of water-soluble cobalt (II) cations in potassium chloride electrolyte. Cobalt (II) chloride electrodes follow the potential profile of pseudocapacitors, where the electric charge is stored at the redox reaction of $\mathrm{Co}^{2+} \leftrightarrow \mathrm{Co}^{4+}$ during the charge-discharge reaction.

In order to obtain information on the electrical resistance from impedance measurements, Nyquist plots of electrodes are shown in Figure 6(d). The diameter of the semicircle at high frequency is related to the charge transfer between the electrode material and the electrolyte, and the tail slope at low frequency is related to the ionic diffusion rate in the electrolyte. ${ }^{41,42}$ The observation of a single semicircular arc in Nyquist plots $\left(Z^{\prime}\right.$ against $\left.Z^{\prime \prime}\right)$ confirms the presence of bulk effects only. The more vertical the line in the impedance plot, the more closely the supercapacitor behaves as an ideal capacitor. The tail slope was $0.5 \mathrm{k} \Omega$ for cobalt (II) chloride-GQDs and $2.5 \mathrm{k} \Omega$ for GQDs, which indicate that the ion diffusion rate of cobalt (II) chloride-GQDs is higher than that of GQDs. This material, cobalt (II) chloride-GQDs, delivers an efficient ion and electron transport, giving rise to faster kinetics and resulting in high charge-discharge capacities at a current density of $1 \mathrm{~mA} / \mathrm{g}$. Thus, the relatively high specific capacitance of cobalt (II) chloride-GQDs might be attributed to the high conductance of GQDs. In this study, the doped cobalt (II) chloride-GQDs sample does not show a semicircle and shows superior rate performance for doped GQDs. These results show that the obtained high specific capacitance promises better electrode performance for practical applications.

\section{Conclusion}

It was demonstrated, for the first time in the literature, that watersoluble cobalt (II) chloride-GQD electrodes can show a reversible redox reaction of $\mathrm{Co}^{2+} \leftrightarrow \mathrm{Co}^{4+}$ with specific capacitance of $300 \mathrm{~F} / \mathrm{g}$, in which a facile hydrothermal method was used for the synthesis of cobalt (II) chloride-GQDs from GO. Interestingly, FTIR, Raman and PL spectroscopic measurements were found to impact strongly the doping process to form cobalt (II) chloride-GQDs. The electrochemical results suggest that properly doped cobalt (II) chloride-GQDs will lead to improvement in the design of high-capacitance pseudocapacitors. The water-soluble cobalt (II) chloride-GQD electrodes can extend the understanding of such a new active cation charge storage mechanism to achieve higher capacitance pseudocapacitors in the near future.

\section{Acknowledgements}

This work was financially supported by Dr DSKPDF, University Grants Commission, India. The authors thank Nishant Kharangarh and Aruna Sharma for useful discussions.

\section{REFERENCES}

1. Miller JR and Simon P (2008) Materials science: electrochemical capacitors for energy management. Science 321(5889): 651-652.

2. Simon $P$ and Gogotsi $Y$ (2008) Materials for electrochemical capacitors. Nature Materials 7(11): 845-854.

3. Liu C, Li F, Ma LP and Cheng HM (2010) Advanced materials for energy storage. Advanced Materials 22(8): E28-E62.

4. Xu C, Xu B, Gu Y et al. (2013) Graphene-based electrodes for electrochemical energy storage. Energy \& Environmental Science 6(5): 1388-1414.

5. Liu W, Feng Y, Yan X, Chen J and Xue Q (2013) Superior microsupercapacitors based on graphene quantum dots. Advanced Functional Materials 23(33): 4111-4122.

6. Gao W (2011) Direct laser writing of micro-supercapacitors on hydrated graphite oxide films. Nature Nanotechnology 6(8): 496-500.

7. El-Kady MF, Strong V, Dubin S and Kaner RB (2012) Laser scribing of high-performance and flexible graphene-based electrochemical capacitors. Science 335(6074): 1326-1330.

8. Zhang S, Li Y, Song $\mathrm{H}$ et al. (2015) Graphene quantum dots as the electrolyte for solid state supercapacitors. Scientific Reports 6 : 19292.

9. Jost K, Dion G and Gogotsi Y (2014) Textile energy storage in perspective. Journal of Material Chemistry A 2(28): 10776-10787.

10. Xiong G, Meng C, Reifenberger R, Irazoqui P and Fisher T (2014) A review of graphene-based electrochemical microsupercapacitors. Electroanalysis 26(1): 30-51.

11. Li Y, Hu Y, Zhao Y et al. (2011) An electrochemical avenue to greenluminescent graphene quantum dots as potential electron-acceptors for photovoltaics. Advanced Materials 23(6): 776-780.

12. Zhang M, Bai L, Shang W et al. (2012) Facile synthesis of water-soluble, highly fluorescent graphene quantum dots as a robust biological label for stem cells. Journal of Materials Chemistry 22(15): 7461-7467.

13. Ji J, Liu J, Lai L et al. (2015) In situ activation of nitrogen-doped graphene anchored on graphite foam for a high-capacity anode. ACS Nano 9(8): 8609-8616.

14. Chen J, Han Y, Kong $X$ et al. (2016) The origin of improved electrical double-layer capacitance by inclusion of topological defects and dopants in graphene for supercapacitors. Angewandte Chemie International Edition 55(44): 13822-13827.

15. Parlak O, Mishra YK, Grigoriev A et al. (2017) Hierarchical aerographite nano-microtubular tetrapodal networks based electrodes as lightweight supercapacitor. Nano Energy 34: 570-577.

16. Mai L, Yang F, Zhao Y et al. (2011) Rational synthesis of silver vanadium oxides/polyaniline triaxial nanowires with enhanced electrochemical property. Nature Communications 11(11): 4992-4996.

17. Zhang $Y$, Sun C, Lu P et al. (2012) Crystallization design of $\mathrm{MnO}_{2}$ towards better supercapacitance. CrystEngComm 14(18): 5892-5897.

18. Lu Z, Chang Z, Zhu W and Sun X (2011) Beta-phased Ni(OH $)_{2}$ nanowall film with reversible capacitance higher than theoretical Faradic capacitance. Chemical Communications 47(34): 9651-9653.

19. Cao L, Xu F, Liang Y and Li H (2004) Preparation of the novel nanocomposite $\mathrm{Co}(\mathrm{OH})_{2}$ /ultra-stable $\mathrm{Y}$ zeolite and its application as a supercapacitor with high energy density. Advanced Materials 16(20): 1853-1857.

20. Chen K, Song S, Li K and Xue D (2013) Water-soluble inorganic salts with ultrahigh specific capacitance: crystallization transformation investigation of $\mathrm{CuCl}_{2}$ electrodes. CrystEngComm 15(47): 10367-10373, http://dx.doi.org/10.1039/C3CE41802B.

21. Hummers WS and Offemann RE (1958) Preparation of graphitic oxide. Journal of the American Chemical Society 80(6): 1339.

22. Kharangarh PR, Kumar A, Sharma RK and Singh G (2017) Thermal effects for the doped graphene quantum dots: cyclic voltammetry. Advanced Materials Proceedings 2(3): 171-175.

23. Kumar R, Agrawal A, Nagarale RK and Sharma A (2016) High-performance supercapacitors from novel metal-doped 
ceria-decorated aminated graphene. Journal of Physical Chemistry $C$ 120(6): 3107-3116.

24. Bard AJ and Faulkner LR (2001) Electrochemical Methods: Fundamentals and Applications, 2nd edn. Wiley New York, NY, USA.

25. Du H, Liu Q, Cheng N et al. (2014) Template-assisted synthesis of CoP nanotubes to efficiently catalyze hydrogen-evolving reaction. Journal of Materials Chemistry A 2(36): 14812-14816.

26. Ye W, Zhang X, Chen Y et al. (2013) Pulsed electrodeposition of reduced graphene oxide on glass carbon electrode as an effective support of electrodeposited Pt microspherical particles: nucleation studies and the application for methanol electro-oxidation. International Journal of Electrochemistry 8: 2122-2139.

27. Chen Y, Wang Q, Zhu C et al. (2012) Graphene/porous cobalt nanocomposite and its noticeable electrochemical hydrogen storage ability at room temperature. Journal of Materials Chemistry 22(13): 5924-5927.

28. Ray SS, Lee MW, Ray SS et al. (2013) Supersonic nanoblowing: a new ultra-stiff phase of nylon 6 in 20-50 nm confinement. Journal of Materials Chemistry C 1(21): 3491-3498.

29. Mattevi C, Eda G, Agnoli S et al. (2009) Evolution of electrical, chemical, and structural properties of transparent and conducting chemically derived graphene thin films. Advanced Functional Materials 19(16): 2577-2583.

30. Pimenta MA, Dresselhaus G, Dresselhaus MS et al. (2007) Studying disorder in graphite-based systems by Raman spectroscopy. Physical Chemistry Chemical Physics 9(11): 1276-1291.

31. Fan Z, Yan J, Zhi L et al. (2010) A three dimensional carbon nanotube/ graphene sandwich and its application as electrode in supercapacitors. Advanced Materials 22: 3723-3728.

32. Yao Y, Xu C, Qin J et al. (2013) Synthesis of magnetic cobalt nanoparticles anchored on graphene nanosheets and catalytic decomposition of orange II. Industrial \& Engineering Chemistry Research 52(49): 17341-17350.

33. Pacile D, Meyer JC, Rodríguez AF et al. (2011) Electronic properties and atomic structure of graphene oxide membranes. Carbon 49(3): 966-972.

34. Li C and Yue Y (2014) Fluorescence spectroscopy of graphene quantum dots: temperature effect at different excitation wavelengths. Nanotechnology 25(43): 435703.

35. Bagri A, Mattevi C, Acik M et al. (2010) Structural evolution during the reduction of chemically derived graphene oxide. Nature Chemistry 2(7): 581-587.

36. Newman R and Chrenko RM (1959) Infrared absorption from L·S splittings in $\mathrm{Co}^{2+}$ salts. Physical Review 115(5): 1147-1152.

37. Ferrari AC and Robertson J (2000) Interpretation of Raman spectra of disordered and amorphous carbon. Physical Review B: Condensed Matter and Materials Physics 61(20): 14095-14107.

38. Zhang Z, Zhang J, Chen N and Qu L (2012) Graphene quantum dots: an emerging material for energy-related applications and beyond. Energy \& Environmental Science 5(10): 8869-8890.

39. Chen $\mathrm{K}$, Yang Y, Li K et al. (2014) $\mathrm{CoCl}_{2}$ designed as excellent pseudocapacitor electrode materials. ACS Sustainable Chemistry \& Engineering 2(3): 440-444.

40. Yan J, Wei T, Fan Z et al. (2010) Preparation of graphene nanosheet/ carbon nanotube/polyaniline composite as electrode material for supercapacitors. Journal of Power Sources 195(9): 3041-3045.

41. Du X, Guo P, Song $H$ and Chen X (2010) Graphene nanosheets as electrode material for electric double-layer capacitors. Electrochimica Acta 55(16): 4812-4819.

42. Portet C, Yushin G and Gogotsi Y (2007) Electrochemical performance of carbon onions, nanodiamonds, carbon black and multiwalled nanotubes in electrical double layer capacitors. Carbon 45(13): 2511-2518.

\section{How can you contribute?}

To discuss this paper, please submit up to 500 words to the journal office at journal@ice.org.uk. Your contribution will be forwarded to the author(s) for a reply and, if considered appropriate by the editor-in-chief, it will be published as a discussion in a future issue of the journal.

ICE Science journals rely entirely on contributions from the field of materials science and engineering. Information about how to submit your paper online is available at www.icevirtuallibrary.com/page/authors, where you will also find detailed author guidelines. 